\title{
Prescribing Trends for the Outpatient Treatment of Adolescents and Young Adults with Type 2 Diabetes Mellitus
}

\author{
Hanna Phan, PharmD, BCPS; Kyle Porter, MAS; \\ Bruce Sill, PharmD, MS; and Milap C. Nahata, PharmD, MS
}

\begin{abstract}
BACKGROUND: Little is known about U.S. outpatient prescribing trends for type 2 diabetes (T2DM) in adolescents and young adults.

OBJECTIVES: To determine (a) trends in the outpatient prescribing of pharmacological and nonpharmacological therapies and (b) factors influencing prescribing trends for adolescents and young adults with T2DM.
\end{abstract}

METHODS: A retrospective, cross-sectional analysis was conducted on office visits of adolescents (12-17 years) and young adults (18-39 years) with T2DM or impaired glucose tolerance (IGT), using the National Ambulatory Medical Care Survey (NAMCS) from 1996-2005. Logistic regression was used to test for prescribing trends over time.

RESULTS: There were an estimated 1.6 million (93.7\% T2DM; 4.4\% T2DM + IGT; 1.9\% IGT) and 22.2 million (88.1\% T2DM; 11.9\% IGT) office visits for adolescents $(0.4 \%$ of all adolescent visits) and young adults $(1.2 \%$ of all young adult visits) associated with T2DM based on ICD-9-CM codes, respectively. In young adults, diabetes drug mentions increased significantly from $39 \%$ of visits with T2DM to $61 \%$ in $2004-2005(P=0.04)$. Oral diabetes medication mentions increased from $20 \%$ to $49 \%(P=0.001)$. However, reports of nonpharmacological therapy decreased from $53 \%$ in $1996-1997$ to $37 \%$ in $2004-2005(P=0.14)$.

CONCLUSIONS: The prescribing of pharmacological treatment for T2DM increased with emphasis on oral agents, while reports of nonpharmacological therapy for T2DM decreased over the 9-year study period with increased use of oral medications in both adolescents and young adults. Health care providers should consistently consider both treatment approaches when prescribing patient care as recommended by treatment guidelines.

J Manag Care Pharm. 2012;18(8):607-14

Copyright $\odot 2012$, Academy of Managed Care Pharmacy. All rights reserved.

\section{What is already known about this subject}

- Type 2 diabetes mellitus (T2DM), once thought of as an illness common to older adults, is now increasing in prevalence among young adults and adolescents.

- Previous studies have examined prescribing trends using private insurance databases for children and adolescents and national ambulatory care databases for adults. From these studies, an increasing trend in use of pharmacological treatment for T2DM has been noted, which is possibly reflective of an increasing incidence of risk factors for T2DM, such as obesity, in the United States.

- Off-label medication use is practiced in the treatment of T2DM in adolescents. A variety of treatment approaches are utilized in young adults and adolescents with T2DM.

\section{What this study adds}

- This study provides data for prescribing trends of pharmacological and nonpharmacological (e.g., lifestyle changes) therapies in the treatment of T2DM for young adults and adolescents.

$\mathrm{T}$ ype 2 diabetes mellitus (T2DM), once thought of as an illness common in older adults, is now increasing in prevalence among young adults and adolescents. ${ }^{1,2}$ According to the Centers for Disease Control and Prevention (CDC), T2DM comprises 90\%-95\% of all diagnosed cases of diabetes. In 2010, diabetes was diagnosed in approximately 18 million people in the United States, with over 200,000 people younger than 20 years of age. ${ }^{1}$ Up to $46 \%$ of all new cases of diabetes among children and adolescents are T2DM. ${ }^{2}$ Although T2DM affects all ethnic groups, it is more commonly diagnosed in nonwhite groups, including American Indian and African American youth. ${ }^{2}$ Among individuals younger than 20 years of age, the overall prevalence of T2DM was 22 per 100,000 or 24.6 per 100,000 person-years. ${ }^{3,4}$ The overall incidence of diabetes has increased over the past decade; this may be attributed in part to an increase in overweight and obese young adults and adolescents in the United States. ${ }^{1-6}$

Diagnosis and treatment of diabetes has evolved over the years. It has been noted that medical nutrition therapy alone in adults with T2DM has not been sufficient for most patients, especially in the first year of diagnosis, resulting in possible need for combination medical nutrition therapy and initial medication therapy ${ }^{7-10}$ As a result, initial treatment using pharmacological therapy, such as metformin, and medical nutrition therapy, such as lifestyle changes in diet and exercise, in adult patients with T2DM is recommended. ${ }^{8,10}$ However, there is a noteworthy difference in the treatment approach for T2DM in adolescents. The American Diabetes Association (ADA) and American Academy of Pediatrics (AAP) suggest that children and adolescents who are not acutely ill at diagnosis can be managed initially using lifestyle changes or nonpharmacological therapy, such as self-management education, diet and nutrition counseling by a dietitian, and exercise or fitness programs. However, when nonpharmacological therapy ceases to meet treatment goals, medication therapy (i.e., metformin) should be initiated. ${ }^{7,11,12}$ Similar to many medications used in the pediatric population, drug therapy approved for treatment of T2DM 
in adolescents is limited. Metformin is currently the only oral diabetes agent approved for children and adolescents aged 10 years and older. Insulin, including long-acting insulins, are also approved for use in management of diabetes, both type 1 and 2 , in children and adolescents. ${ }^{11-13}$

Limited data about the trends of pharmacologic treatment of T2DM in young adults and adolescents have been described. ${ }^{13-18}$ However, updates in prescribing trends of medication therapy for T2DM related to payment source (e.g., insurance), prescriber type or specialty, ethnicity, and race are lacking, especially for adolscents.. ${ }^{13-15}$ The influence of such factors as insurance and prescriber type can affect treatment modalities used by providers and thus influence prescribing trends. The introduction of newer drug therapies and changes in treatment guidelines may affect prescribing trends in these patient populations. A study examining prescribing trends for pharmacological and nonpharmacological therapies may stimulate further research to improve medication use and health outcomes in young adults and adolescents with T2DM.

The objectives of this study were to determine prescribing trends of pharmacological and nonpharmalogical therapies for the management of T2DM in adolescents (12-17 years) and young adults (18-39 years) and factors influencing those prescribing trends in U.S. outpatient settings from 1996-2005.

\section{Methods}

This was a retrospective, cross-sectional study utilizing the National Ambulatory Medical Care Survey (NAMCS) database from 1996 to 2005 and was approved by the Ohio State University Institutional Review Board. This study examined trends in physician prescribing of medications and nonpharmacological therapies for T2DM in adolescents and young adults. NAMCS is a national probability sample survey conducted by the Division of Health Care Statistics of the National Center for Health Statistics (NCHS) and the CDC. The basic sampling unit for NAMCS is the physician-patient encounter or office visit, defined as a visit to a nonfederally employed office-based, direct patient care physician (excluding those in the specialties of radiology, anesthesiology, and pathology). Data collected from the medical records of each sampled office visit included patient sociodemographics, physician specialty, reason(s) for the visit, source of payment, diagnoses, medication records, nonspharmacological treatments or interventions (NAMCS variables DIETNUTR and EXERCISE), and type of insurance coverage. Based on the multistage sampling design, each office visit is assigned a statistical sampling weight. The target estimation population is all ambulatory office visits in the United States. Additional description of the methods used in NAMCS sampling, weighing, data processing, and quality control is available for public review. ${ }^{19}$

Office visits with adolescents (12-17 years) and young adults (18-39 years) having a diagnosis of T2DM were identified using
International Classification of Disease, Ninth Revision, Clinical Modification (ICD-9-CM) codes from the visit record of the NAMCS dataset (Appendix A and B). An indicator variable for T2DM in patients aged 12 to 39 years was created and given the value of 1 when such a visit was identified. Not included in this identification were office visits of patients aged less than 12 years, those patients who presented with hyperglycemia without an ICD-9-CM code for T2DM or impaired glucose tolerance (IGT), or those patients who had type 1 diabetes mellitus. In addition, the presence of associated comorbidities, such as obesity, nephropathy, neuropathy, peripheral vascular disorders, hypertension, and hyperlipidemia were identified by ICD-9-CM code. Specific drug therapy was also identified using the NAMCS coding system and was grouped in therapeutic categories (Appendix C).

\section{Statistical Analysis}

All analyses were conducted using STATA statistical software version 10.1. Estimation and analysis procedures followed the guidelines provided by the CDC in the document "Using Ultimate Cluster Models with NAMCS and NHAMCS Public Use Files." ${ }^{19}$ For data files prior to 2002, the CSTRATM and CPSUM variables were created to allow for variance estimation, assuming ultimate cluster design in STATA (CSTRATM and CPSUM were included starting in 2002). STATA survey set properties were set to pweight $=$ PATWT, strata $=$ CSTRATM, and psu $=$ CPSUM. The sampling weights were designed to be nationally representative of all office visits. Estimates with a relative standard error greater than 30\% were considered unreliable and not reported per NAMCS standards.

Separate analyses were performed for adolescents (aged 12-17 years) and young adults (aged 18-39 years) with T2DM. All data presented were weighted. Descriptive statistics for patient and physician characteristics were calculated as percentages of visits for the entire 1996-2005 cohort and also separately for each major treatment category: medication alone, nonpharmacological therapy alone (diet and/or exercise therapy), combination therapy (medication plus nonpharmacological), and no treatment. The number of visits with drug mentions from Appendix $C$ and nonpharmacological therapy mentions were estimated for each age group. For the aged 18-39 cohort, drug mention estimates were also made separately for oral medications and insulin or insulin analogues. In the aged 12-17 cohort, 5-year periods were required to obtain reliable estimates. Chi-square tests were performed to test for differences between 1996-2000 and 2001-2005. For the aged 18-39 cohort, estimates were obtained for 2-year periods; linear trends were tested using logistic regression with time as the independent variable for each outcome. Sampling weights were divided by the number of years in each period to account for combining results across survey years. 


\section{Results}

\section{Overall Patterns of Ambulatory Care \\ Visits Associated with T2DM}

Between 1996 and 2005, the estimated total number of office visits in the United States was 426 million for adolescents (12-17 years) and 1.95 billion for young adults (18-39 years). The estimated number of visits by patients with diagnoses of T2DM or IGT was 1.6 million for adolescents and 22.2 million for young adults during this time period. Among adolescents, $93.7 \%$ of the 1.6 million visits were classified as T2DM without IGT; estimates of visits involving IGT were not reliable. For young adults, $88.1 \%$ of the 22.2 million visits were classified as T2DM alone, and $11.9 \%$ were IGT alone.

A majority of adolescent patients were non-Hispanic (75\%), white $(81 \%)$, and from the southern U.S. geographic region (49\%). There were an equal percentage of male and female patients. Most of the adolescent patients were reported to have used private insurance (64\%). Pediatricians (38\%) and general/ family medicine $(32 \%)$ specialties were the most common physicians among adolescent office visits reported. Other demographic estimates did not meet NAMCS reliability criteria.

Among the young adults patient group, most patients were white $(73 \%)$, and $85 \%$ of patients with a declared ethnicity were non-Hispanic. Female patients represented the majority (61\%), and two-thirds of the total population was between 31-39 years of age (68\%). The most represented geographic region was the southern region of the United States (39\%). Hypertension was the most commonly reported comorbidity (13\%), followed by obesity (8\%). Private insurance (66\%) was the most common payment source, followed by Medicare/Medicaid (19\%). Other unlisted specialties (35\%) were the most common specialty reported in this patient group, followed by general/family medicine (33\%) and internal medicine (26\%).

\section{Trends for Pharmacological and Nonpharmacological Treatment of T2DM}

Proportions of patient visits associated with T2DM or IGT that did not report either prescribed medication or nonpharmacological intervention (e.g., diet and exercise) were similar between adolescents and young adults, 33\% and $31.5 \%$, respectively. Use of combination pharmacological and nonpharmacological therapy was slightly higher for adolescents (31\%) compared with young adults (25.7\%), whereas use of either treatment modality alone was more common in young adults (Table 1).

Of visits from adolescents with T2DM from 1996-2000, $43 \%$ (confidence interval $[\mathrm{CI}]=24 \%-65 \%$ ) had a mention of at least 1 diabetes medication, and 58\% (CI=37\%-77\%) reported nonpharmacological intervention. From 2001-2005, 59\% $(\mathrm{CI}=26 \%-86 \%)$ had drug mentions, and 32\% ( $\mathrm{CI}=12 \%-68 \%)$ reported nonpharmacological intervention. Neither change was statistically significant.
For the young adult group, the percentage of visits for T2DM with a drug mention increased significantly from 39\% in 1996 1997 to $61 \%$ in $2004-2005$ ( $P=0.04$; Table 2). Specifically, the percentage of visits with oral medication mentions increased from $20 \%$ to $49 \%(P=0.001)$. Reports of nonpharmacological therapy decreased from 53\% in 1996-1997 to 37\% in 2004$2005(P=0.14)$.

\section{Discussion}

Much of the adult data regarding U.S. prescribing trends for the outpatient treatment of T2DM are for older adults, so there are limited data for young adults. ${ }^{16,17}$ Skaer et al. (2006) examined payment sources from 1990-2001 and found that access to drug therapy was associated with source of insurance coverage. ${ }^{16}$ Since this study, there has remained a lack of data describing prescribing trends based on such factors as payment source, health care provider, and geographic location in both the young adult and adolescent populations. In a study covering a 3-year period (1997-2000), Cohen et al. (2003) found an increasing prescribing trend of combination oral agents versus monotherapy, including insulin. ${ }^{17}$ In our study, we found a significant trend of increased oral medication use; however, we did not find a change in use of insulin therapy. The Cohen et al. study, which examined data from privately insured U.S. patients, lacked data regarding those patients with different coverage such as Medicaid. ${ }^{17}$

There are limited studies describing T2DM therapy trends in adolescents..$^{14,15}$ Liberman et al. (2009) examined trends in use of medications for antihypertensive, antidiabetic, and dyslipidemic in children and adolescents and found an increased use of oral diabetes therapy by approximately $15 \%$ over a 3 -year time period..$^{14}$ Similarly, Cox et al. (2008), in a study of chronic medication use trends, found doubled use of diabetes medications over a 3 -year period. ${ }^{15}$ However, these studies utilized private insurance databases, which lacked representation from other populations, including Medicaid and self-pay patients. ${ }^{14,15}$ Off-label medication use is also a distinct characteristic in the treatment of adolescents given the limited availability of approved agents. Compared with the years 19962000 , off-label medication use may be a part of the increased trend seen in the years 2001-2005, as newer agents became available on the market. However, there are various factors that may have affected change in therapy use over the years, including those related to prescribers (e.g., familiarity with agents) and patients (e.g., severity of disease).

Examination of the trends in the use of nonpharmacological therapy for T2DM was limited in previous studies. ${ }^{14-17}$ Our study identified changes in prescribing trends of outpatient medications and nonpharmacological therapy, such as diet and/or exercise. Interestingly, during the study period, drug therapy utilization use increased and use of nonpharmacological therapy decreased. However, combination of 
Prescribing Trends for the Outpatient Treatment of

Adolescents and Young Adults with Type 2 Diabetes Mellitus

\section{TABLE 1 Descriptive Statistics Overall and By Therapy Category for} Young Adults (18-39 Years) with Type 2 Diabetes, 1996-2005

\begin{tabular}{|c|c|c|c|c|c|c|c|c|c|c|}
\hline \multirow{3}{*}{$\begin{array}{l}\text { Characteristic } \\
\text { Weighted number of visits }(1,000,000 \mathrm{~s})\end{array}$} & \multicolumn{10}{|c|}{ Weighted Percentage of Visits $(95 \% \mathrm{CI})^{\mathrm{a}}$} \\
\hline & \multicolumn{2}{|c|}{ All Visits } & \multicolumn{2}{|c|}{ Medication Alone } & \multicolumn{2}{|c|}{ Med + Nonpharmb } & \multicolumn{2}{|c|}{ Nonpharm Alone $e^{c}$} & \multicolumn{2}{|c|}{ No Treatment } \\
\hline & 22.5 & $(19.7-25.3)$ & 5.1 & $(3.9-6.3)$ & 5.8 & $(4.5-7.1)$ & 4.5 & $(3.3-5.6)$ & 7.1 & $(5.7-8.6)$ \\
\hline \multicolumn{11}{|l|}{ Age } \\
\hline $18-25$ & 13 & $(10-16)$ & 12 & $(6-21)$ & 8 & $(4-14)$ & 12 & $(7-21)$ & 18 & $(12-26)$ \\
\hline $25-30$ & 20 & $(13-30)$ & 19 & $(12-27)$ & 19 & $(13-28)$ & 23 & $(15-34)$ & 16 & $(10-25)$ \\
\hline $31-39$ & 68 & $(63-73)$ & 68 & $(57-77)$ & 73 & $(64-81)$ & 64 & $(52-75)$ & 66 & $(56-74)$ \\
\hline \multicolumn{11}{|l|}{ Gender } \\
\hline Female & 61 & $(56-67)$ & 50 & $(38-62)$ & 64 & $(53-74)$ & 63 & $(51-73)$ & 67 & $(57-75)$ \\
\hline \multicolumn{11}{|l|}{ Race } \\
\hline White & 73 & $(67-78)$ & 76 & $(62-86)$ & 70 & $(58-80)$ & 73 & $(61-83)$ & 73 & $(63-81)$ \\
\hline Black & 20 & $(16-26)$ & 16 & $(9-27)$ & 22 & $(14-34)$ & 20 & $(12-31)$ & 21 & $(14-30)$ \\
\hline Asian/Pacific Islander & 7 & $(4-11)$ & & NR & & NR & & NR & & NR \\
\hline Other/Unknown & & NR & & NR & & NR & & NR & & NR \\
\hline \multicolumn{11}{|l|}{ Ethnicity } \\
\hline Hispanic & 11 & $(7-15)$ & & NR & & NR & 17 & $(10-28)$ & & NR \\
\hline Non-Hispanic & 63 & $(57-68)$ & 64 & $(52-74)$ & 71 & $(61-80)$ & 53 & $(41-64)$ & 61 & $(51-71)$ \\
\hline Undeclared & 27 & $(22-32)$ & 26 & $(17-38)$ & 22 & $(14-32)$ & 30 & $(20-43)$ & 29 & $(20-39)$ \\
\hline \multicolumn{11}{|l|}{ Comorbidities } \\
\hline Obesity/overweight & 8 & $(5-11)$ & & NR & 14 & $(9-23)$ & & NR & & NR \\
\hline Hypertension & 13 & $(10-17)$ & 19 & $(11-31)$ & 20 & $(13-30)$ & & NR & & NR \\
\hline Hyperlipidemia & 5 & $(3-7)$ & & NR & & NR & & NR & & NR \\
\hline Nephropathy & & NR & & NR & & NR & & NR & & NR \\
\hline Retinopathy & 3 & $(2-5)$ & & NR & & NR & & NR & 9 & $(6-15)$ \\
\hline Diabetic neuropathy & & NR & & NR & & NR & & NR & & NR \\
\hline Peripheral vascular disorder & & NR & & NR & & NR & & NR & & NR \\
\hline \multicolumn{11}{|l|}{ Payment source } \\
\hline Private insurance & 66 & $(60-71)$ & 67 & $(57-77)$ & 70 & $(59-79)$ & 63 & $(51-74)$ & 63 & $(54-72)$ \\
\hline Medicare/Medicaid/WC & 19 & $(16-23)$ & 15 & $(9-23)$ & 15 & $(10-23)$ & 23 & $(14-35)$ & 23 & $(17-32)$ \\
\hline Self-pay & 7 & $(5-10)$ & & NR & & NR & & NR & & NR \\
\hline Other/unknown & 8 & $(6-12)$ & & NR & & NR & & NR & & NR \\
\hline \multicolumn{11}{|l|}{ Physician specialty } \\
\hline Endocrinologist & \multicolumn{2}{|r|}{ NR } & \multicolumn{2}{|r|}{ NR } & \multicolumn{2}{|c|}{ NR } & \multicolumn{2}{|r|}{ NR } & \multicolumn{2}{|c|}{ NR } \\
\hline General/family medicine & 33 & $(28-39)$ & 37 & $(26-49)$ & 46 & $(36-57)$ & 34 & $(24-46)$ & 18 & $(12-27)$ \\
\hline Internal medicine & 26 & $(20-31)$ & 36 & $(24-49)$ & 25 & $(17-36)$ & 22 & $(13-35)$ & 21 & $(13-31)$ \\
\hline Other specialties & 35 & $(30-41)$ & 19 & $(12-28)$ & 23 & $(16-32)$ & 40 & $(29-52)$ & 54 & $(44-64)$ \\
\hline \multicolumn{11}{|l|}{ Region } \\
\hline Northeast & 14 & $(10-18)$ & & NR & 9 & $(5-15)$ & 17 & $(10-28)$ & 16 & $(10-24)$ \\
\hline Midwest & 24 & $(19-30)$ & 29 & $(19-41)$ & 28 & $(19-39)$ & 26 & $(17-37)$ & 17 & $(11-25)$ \\
\hline South & 39 & $(33-46)$ & 38 & $(27-51)$ & 38 & $(28-50)$ & 41 & $(29-54)$ & 40 & $(31-51)$ \\
\hline West & 23 & $(18-29)$ & 21 & $(13-31)$ & 25 & $(16-36)$ & & NR & 27 & $(19-3)$ \\
\hline \multicolumn{11}{|c|}{$\begin{array}{l}\text { a Except first row, which is weighted frequency. } \\
b_{\text {Medication plus nonpharmacological therapy. }} \\
{ }^{c} \text { Nonpharmacological therapy. } \\
C I=\text { confidence interval; NR }=\text { Not reliable, relative standard error }>30 \% ; W C=\text { worker's compensation. }\end{array}$} \\
\hline
\end{tabular}

pharmacological and nonpharamcological therapy was found to be greater among adolescents compared with young adults. This may reflect practices emphasizing medical intervention, such as an oral diabetes medications as per adult guidelines, compared with pediatric guidelines in which nutritional medical therapy is considered first line, initial treatment. ${ }^{7,8,11,12}$ The greater use of nonpharmacological therapy among adolescents may also be a result of limited approved agents for this age population. Lack of reported nonpharmacological therapy in both groups may also be attributed to under-reporting, as such approaches may not have been considered traditional medical treatment for T2DM, compared with drug therapies, by survey data providers.

Patient factors such as race and ethnicity, provider type, 


\section{Prescribing Trends for the Outpatient Treatment of Adolescents and Young Adults with Type 2 Diabetes Mellitus}

\section{TABLE 2 Medication Trends in Young Adults (18-39 Years) with Type 2 Diabetes, 1996-2005}

\begin{tabular}{|c|c|c|c|c|c|}
\hline \multirow[b]{2}{*}{ Treatment } & \multicolumn{5}{|c|}{ Percentage of Visits with Patient Taking the Indicated Medication $(95 \% \mathrm{CI})^{\mathrm{a}}$} \\
\hline & 1996-1997 & 1998-1999 & $2000-2001$ & $2002-2003$ & $2004-2005$ \\
\hline Any diabetes medication ${ }^{\mathrm{b}}$ & $\begin{array}{c}0.8(0.6-1.0) \\
39(28-51)\end{array}$ & $\begin{array}{c}0.9(0.6-1.2) \\
50(38-62)\end{array}$ & $\begin{array}{c}1.2(0.9-1.6) \\
44(32-57)\end{array}$ & $\begin{array}{c}1.2(0.9-1.7) \\
49(36-61)\end{array}$ & $\begin{array}{c}1.3(1.0-1.8) \\
61(46-74)\end{array}$ \\
\hline Oral diabetes medication ${ }^{\mathrm{c}}$ & $\begin{array}{c}0.3(0.2-0.5) \\
20(12-32)\end{array}$ & $\begin{array}{c}0.4(0.3-0.6) \\
32(22-43)\end{array}$ & $\begin{array}{c}0.6(0.5-0.9) \\
32(21-44)\end{array}$ & $\begin{array}{c}0.7(0.5-0.9) \\
38(26-52)\end{array}$ & $\begin{array}{c}0.9(0.7-1.3) \\
49(35-63)\end{array}$ \\
\hline Insulin/insulin analogs & $\begin{array}{c}0.5(0.3-0.7) \\
19(13-28)\end{array}$ & $\begin{array}{c}0.5(0.3-0.7) \\
20(11-34)\end{array}$ & $\begin{array}{c}0.7(0.4-1.1) \\
18(11-28)\end{array}$ & $\begin{array}{c}0.7(0.4-1.0) \\
18(11-28)\end{array}$ & $\begin{array}{c}0.5(0.3-0.7) \\
16(9-27)\end{array}$ \\
\hline Nonpharmacological therapy & $\begin{array}{l}16(15-18)^{\mathrm{d}} \\
53(42-64) \\
\end{array}$ & $\begin{array}{l}16(14-18)^{\mathrm{d}} \\
41(30-54) \\
\end{array}$ & $\begin{array}{l}14(12-16)^{d} \\
51(39-63)\end{array}$ & $\begin{array}{l}17(15-19)^{\mathrm{d}} \\
45(34-57) \\
\end{array}$ & $\begin{array}{l}15(13-17)^{\mathrm{d}} \\
37(25-51) \\
\end{array}$ \\
\hline \multicolumn{6}{|c|}{ 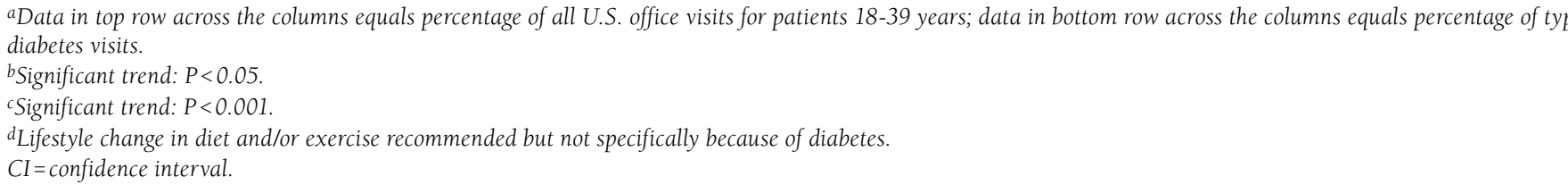 } \\
\hline
\end{tabular}

and geographic location of subjects were also examined in this study. In our study, race and ethnicity were similar to previous studies with mostly white, non-Hispanic patients. Health care provider and geographic location were factors lacking in previous studies among both age populations. ${ }^{14,15,17}$ From a health care provider perspective, general pediatricians and family medicine providers were most common for adolescents in this group, which may indicate the treatment of T2DM occurred in general practices versus managed by specialists. Conversely, unlisted specialists were the majority of providers for young adults. Most of the young adult and adolescent populations used in this study were from the southern U.S. geographic region. Information regarding the most common providers, geographic location, and the prescribing trend of increased pharmacological and decreased nonpharmacological therapy use can help guide education for providers for appropriate utilization of both modalities in the management of T2DM in young adults and adolescents.

\section{Limitations}

Although this study has provided useful information regarding patient- and prescriber-specific trends in a once-overlooked population of T2DM patients, limitations should be considered. Since the basic unit of measure in the NAMCS database is a single office visit, therapeutic outcomes, such as hemoglobin Alc values, were not available. Also, this database does not provide information about patient follow-up, interventions, and medication adherence. The database is limited to 6 medications per visit; this may lead to possible missed observations in reported drug therapy, although the vast majority of patients are not likely to receive more than 6 medications. The database revealed a limited patient sample of adolescents with T2DM, which may be attributed to under-reporting of data in this patient population. Also noteworthy is the limitations in data span through 2005; patterns do not reflect more recent prescribing trends such as the use of incretins. Although the study period ended in 2005, vast majorities of data are still valid as there are limited data that describe prescribing trends of T2DM therapies in younger populations. Although some new medications have come on the market, none have been labeled for pediatric (and thus adolescent) use since 2005.

\section{Conclusions}

Over the study period, it was found that patients were prescribed a variety of diabetes medications, including oral and insulin therapies, as well as nonpharmacologial therapy, with trends changing as a result of evolving treatment guidelines and marketing of newer agents. This study expands the knowledge of prescribing trends of T2DM medications and nonpharmacological therapy and patient-specific factors such as health care providers prescribing therapies, geographic location, and payment provider. With our findings of an increased trend of pharmacological therapy paired with a decreased trend of nonpharmacological therapy, interventions such as additional education to providers should be considered. Such education may encourage optimal and consistent use of both treatment types in the care of young adults and adolescents as recommended by treatment guidelines. Future studies on prescribing trends, especially among younger populations (e.g., adolescents), are needed with the continued introduction of newer oral and injectable diabetes agents for the treatment of T2DM where approved medications are limited. 


\section{Authors}

HANNA PHAN, PharmD, BCPS, is Assistant Professor, Department of Pharmacy Practice and Science, College of Pharmacy, and Assistant Professor, Department of Pediatrics, College of Medicine, The University of Arizona, Tucson, Arizona. KYLE PORTER, MAS, is Senior Consulting Research Statistician, Center for Biostatistics, and MILAP C. NAHATA, PharmD, MS, is Professor and Division Chair, Division of Pharmacy Practice and Administration, College of Pharmacy and Medicine, Department of Internal Medicine and Pediatrics, College of Medicine, The Ohio State University, Columbus, Ohio. BRUCE SILL, PharmD, MS, is Executive Manager, Medical External Affairs, Clinical Science \& Outcomes, Takeda Pharmaceuticals U.S.A., Deerfield, Illinois.

AUTHOR CORRESPONDENCE: Milap C. Nahata, PharmD, MS, The Ohio State University College of Pharmacy, 500 W. 12th Ave., Columbus, OH 43210. Tel: 614-292-2472;

E-mail: nahata.1@osu.edu.

\section{DISCLOSURES}

This study was funded by a grant from Takeda Pharmaceuticals U.S.A., Deerfield, Illinois. Portions of this study were presented at the Academy of Managed Care Pharmacy Educational Conference, October 7-9, 2009, San Antonio, Texas. Concept and design were contributed primarily by Nahata and Phan, with input from Sill and Porter. Phan and Porter had primary responsibility for data collection; data interpretation was the work of Nahata and Phan, with input from Porter and Sill. The manuscript was primarily written by Phan, with assistance from Nahata, Porter, and Sill; its revision was primarily the work of Phan, with assistance from Porter, Nahata, and Sill.

\section{REFERENCES}

1. Centers for Disease Control and Prevention. National diabetes fact sheet: national estimates and general information on diabetes and prediabetes in the United States, 2011. Atlanta, GA: U.S. Department of Health and Human Services, Centers for Disease Control and Prevention, 2011. Available at: http://www.cdc.gov/diabetes/pubs/pdf/ndfs_2011.pdf. Accessed September 23, 2012.

2. Centers for Disease Control and Prevention. Diabetes public health resource: children and diabetes. April 3, 2012. Available at: http://www.cdc. gov/diabetes/projects/cda2.htm. Accessed September 23, 2012.

3. SEARCH for Diabetes in Youth Study Group, Liese AD, D'Agostino RB Jr, et al. The burden of diabetes mellitus among US youth: prevalence estimates from the SEARCH for Diabetes in Youth Study. Pediatrics. 2006;118(4):1510-18.

4. Writing Group for the SEARCH for Diabetes in Youth Study Group, Dabelea D, Bell RA, D'Agostino RB Jr, et al. Incidence of diabetes in youth in the United States. JAMA. 2007;297(24):2716-24.

5. Ogden CL, Carroll MD. Prevalence of overweight, obesity, and extreme obesity among adults: United States, trends 1960-1962 through 2007-2008 Centers for Disease Control and Prevention, National Center for Health Statistics. June 2010. Available at: http://www.cdc.gov/NCHS/data/hestat/obesity_adult_07_08/obesity_adult_07_08.pdf. Accessed September 23, 2012.
6. Ogden CL, Carroll MD. Prevalence of obesity among children and adolescents: United States, trends 1963-1965 through 2007-2008. Centers for Disease Control and Prevention, National Center for Health Statistics. June 2010. Available at: http://www.cdc.gov/nchs/data/hestat/obesity_ child_07_08/obesity_child_07_08.pdf. Accessed September 23, 2012.

7. Qaseem A, Humphrey LL, Sweet DE, Starkey M, Shekelle P; Clinical Guidelines Committee of the American College of Physicians. Oral pharmacologic treatment of type 2 diabetes mellitus: a clinical practice guideline from the American College of Physicians. Ann Intern Med. 2012;156(3):218-31

8. American Diabetes Association. Standards of medical care in diabetes2010. Diabetes Care. 2010;33(Suppl 1):S11-S61.

9. Nathan DM, Buse JB, Davidson MB, et al. Medical management of hyperglycemia in type 2 diabetes: a consensus algorithm for the initiation and adjustment of therapy: a consensus statement from the American Diabetes Association and European Association for the Study of Diabetes. Diabetes Care. 2009;32(1):193-203.

10. American Diabetes Association. Standards of medical care in diabetes-2012. Diabetes Care. 2012;35(Suppl 1):S11-S63.

11. Gahagan S, Silverstein J, American Academy of Pediatrics Committee on Native American Child Health, American Academy of Pediatrics Section on Endocrinology. Prevention and treatment of type 2 diabetes mellitus in children, with special emphasis on American Indian and Alaska Native children. American Academy of Pediatrics Committee on Native American Child Health. Pediatrics. 2003;112(4):e328-e347.

12. No authors listed. Type 2 diabetes in children and adolescents. American Diabetes Association. Diabetes Care. 2000;23(3):381-89.

13. Rosenbloom AL. Increasing incidence of type 2 diabetes in children and adolescents: treatment considerations. Pediatr Drugs. 2002;4(4):209-21.

14. Liberman JN, Berger JE, Lewis M. Prevalence of antihypertensive, antidiabetic, and dyslipidemic prescription medication use among children and adolescents. Arch Pediatr Adolesc Med. 2009;163(4):357-64.

15. Cox ER, Halloran DR, Homan SM, Welliver S, Mager DE. Trends in the prevalence of chronic medication use in children: 2002-2005. Pediatrics. 2008;122(5):e1053-61.

16. Skaer TL, Sclar DA, Robison LM. Trends in the prescribing of oral agents for the management of type 2 diabetes mellitus in the United States, 19902001: does type of insurance influence access to innovation? Diabetes Educ. 2006;32(6):940-53.

17. Cohen FJ, Neslusan CA, Conklin JE, Song Z. Recent antihyperglycemic prescribing trends for U.S. privately insured patients with type 2 diabetes. Diabetes Care. 2003;26(6):1847-51

18. Centers for Disease Control and Prevention, National Center for Health Statistics. Ambulatory health care data. Questionnaires, datasets, and related documentation. Downloadable documentation. NAMCS, 1993-2010. [On-line]. September 6, 2012. Available at: http://www.cdc.gov/nchs/ahcd/ ahcd_questionnaires.htm\#documentation. Accessed September 28, 2012.

19. Centers for Disease Control and Prevention, National Center for Health Statistics. Using ultimate cluster models with NAMCS and NHAMCS public use files. Ambulatory Statistics Branch. September 28, 2004. Available at: http://www.cdc.gov/nchs/data/ahcd/ultimatecluster.pdf. Accessed September 23, 2012. 
Prescribing Trends for the Outpatient Treatment of

Adolescents and Young Adults with Type 2 Diabetes Mellitus

\section{APPENDIX A List of ICD-9-CM Codes for Patients with Type 2 Diabetes Mellitus} and Impaired Glucose Tolerance and Possible Comorbidities

\begin{tabular}{l|l}
\hline ICD-9-CM Code & \multicolumn{1}{c}{ Diagnoses } \\
\hline 790.21 & Impaired fasting glucose \\
\hline 790.22 & Impaired glucose tolerance test (oral); elevated glucose tolerance test \\
\hline 790.29 & Other abnormal glucose \\
\hline 250.00 & Type II diabetes mellitus without mention of complication, or unspecified type, not stated as uncontrolled \\
\hline 250.02 & Type II diabetes mellitus without mention of complication, uncontrolled or unspecified type \\
\hline 250.40 & Type II diabetes mellitus with renal manifestations, or unspecified type, not stated as uncontrolled \\
\hline 250.42 & Type II diabetes mellitus with renal manifestations, uncontrolled \\
\hline 250.50 & Type II diabetes mellitus with ophthalmic manifestations, or unspecified type, not stated as uncontrolled \\
\hline 250.52 & Type II diabetes mellitus with ophthalmic manifestations, uncontrolled \\
\hline 250.62 & Type II diabetes mellitus with neurological manifestations, or unspecified type, not stated as uncontrolled \\
\hline 250.70 & Type II diabetes mellitus with neurological manifestations, uncontrolled \\
\hline 250.72 & Type II diabetes mellitus with peripheral circulatory disorders, or unspecified type, not stated as uncontrolled \\
\hline 250.80 & Type II diabetes mellitus with peripheral circulatory disorders, uncontrolled \\
\hline 250.82 & $\begin{array}{l}\text { Type II diabetes mellitus with other specified manifestations, or unspecified type, not stated as uncontrolled; diabetic hypoglycemia; } \\
\text { hypoglycemic shock }\end{array}$ \\
\hline 250.90 & Type II diabetes mellitus with other specified manifestations, uncontrolled; diabetic hypoglycemia; hypoglycemic shock \\
\hline 250.92 & Type II diabetes mellitus with unspecified complication, or unspecified type, not stated as uncontrolled \\
\hline ICD-9-CM = International Classification of Diseases, Ninth Revision, Clinical Modification.
\end{tabular}

\section{APPENDIX B List of ICD-9-CM Codes for} Possible Comorbidities

\begin{tabular}{l|l}
\hline ICD-9-CM Code & \multicolumn{1}{c}{ Diagnoses } \\
\hline 272.4 & Other and unspecified hyperlipidemia \\
\hline 401.9 & Unspecified essential hypertension \\
\hline 278.00 & Obesity, unspecified \\
\hline 278.01 & Morbid obesity; severe obesity \\
\hline 278.02 & Overweight \\
\hline
\end{tabular}

ICD-9-CM = International Classification of Diseases, Ninth Revision, Clinical Modification. 
Prescribing Trends for the Outpatient Treatment of Adolescents and Young Adults with Type 2 Diabetes Mellitus

\section{APPENDIX C Medications Used for Type 2 Diabetes Mellitus and NAMCS Medication Codes}

\begin{tabular}{l|l|l}
\hline Medications & \multicolumn{1}{|c|}{ Therapeutic Category } & \multicolumn{1}{c}{ NAMCS Medication Codes } \\
\hline Metformin & Metformin & $95111,95133,02189,02286,02302,04113,04175$ \\
\hline Pioglitazone & Thiazolidinedione & 02311,99090 \\
\hline Rosiglitazone & Thiazolidinedione & 00107,99030 \\
\hline Chlorpropamide & Sulfonylurea & 06625,09250 \\
\hline Glipizide & Sulfonylurea & $01037,13553,91081,05106$ \\
\hline Glyburide & Sulfonylurea & $03016,40580,50035,93069,93305$ \\
\hline Glimepride & Sulfonylurea & 96138,99056 \\
\hline Unspecified sulfonylurea & Sulfonylurea & 93150 \\
\hline Repaglinide & Meglitinide & 01042,98101 \\
\hline Nateglinide & Meglitinide & $01076,04674,20380,06152$ \\
\hline Acarbose & Alpha-glucosidase inhibitors & 96058,20232 \\
\hline Miglitol & Alpha-glucosidase inhibitors & 00038 \\
\hline Glyburide + metformin & Fixed dose combination oral agent & 00209 \\
\hline Glipizide + metformin & Fixed dose combination oral agent & 03181 \\
\hline Pioglitazone + metformin & Fixed dose combination oral agent & 06061 \\
\hline Rosiglitazone + metformin & Fixed dose combination oral agent & 03105 \\
\hline Insulin, insulin analogs & Injectable & $00487,03273,14412,14727,15475,15678,15680,16003,17303,17304,19568$, \\
& & $19648,25598,27748,33073,33078,33808,35575,35576,40815,41380,41855$, \\
& & $41895,42515,60725,60730,61045,92045,92046,92101,92102,94116,95010$, \\
\hline Pramlintide & Injectable & $06053,95075,97017,00253,01267,02209,06164,01214,01266,03306$ \\
\hline Exenatide & Injectable & 05162 \\
\hline NAMCS Nation
\end{tabular}

NAMCS = National Ambulatory Medical Care Survey. 